\title{
Flagellar incorporation of proteins follows at least two different routes in
}

\section{trypanosomes}

Laetitia Vincensini*†, Thierry Blisnick*, Eloïse Bertiaux*, Sebastian Hutchinson*, Christina Georgikou*, Cher-Pheng Ooi*2 ${ }^{2}$ and Philippe Bastin*1

*Trypanosome Cell Biology Unit, Institut Pasteur \& INSERM U1201, 25 rue du Docteur Roux, 75015 Paris, France

†Present address: Sorbonne Universités, UPMC, INSERM, CNRS, Centre d'Immunologie et des Maladies Infectieuses, U1135, ERL8255, 91 Bd de l'hôpital, 75013 Paris, France

${ }^{2}$ Present address: Department of Life Sciences, Sir Alexander Fleming Building, Imperial College-South Kensington, London SW7 2AZ, United Kingdom

${ }^{1}$ To whom correspondence should be addressed: Philippe Bastin (pbastin@pasteur.fr). Phone xx-33-1-40 613835 .

Keywords: cilia and flagella, axoneme, central pair, radial spoke, outer dynein arms, arginine kinase, organelle assembly

Abbreviations used: EGFP, enhanced green fluorescent protein; IFA, immunofluorescence assay; IFT, intraflagellar transport; RNAi, RNA interference; RSP, radial spoke protein 


\begin{abstract}
Background information. Eukaryotic cilia and flagella are sophisticated organelles composed of several hundreds of proteins that need to be incorporated at the right time and the right place during assembly.

Results. Two methods were used to investigate this process in the model protist Trypanosoma brucei: inducible expression of epitope-tagged labelled proteins and fluorescence recovery after photobleaching (FRAP) of fluorescent fusion proteins. This revealed that skeletal components of the radial spokes (RSP3), the central pair (PF16) and the outer dynein arms (DNAI1) are incorporated at the distal end of the growing flagellum. They display low or even no visible turnover in mature flagella, a finding further confirmed by monitoring a heavy chain of the outer dynein arm. In contrast, the membrane-associated protein arginine kinase 3 (AK3) showed rapid turnover in both growing and mature flagella, without particular polarity and independently of intraflagellar transport.

Significance. These results demonstrate different modes of incorporation for structural and membrane-associated proteins in flagella, indicating their likely reliance on different machinery. The absence of turnover of structural elements supports the view that the length of the mature flagellum in trypanosomes is not modified after assembly.
\end{abstract}




\section{Introduction}

Cilia and flagella are ubiquitous organelles whose architecture is highly conserved, from protists to mammals. The distinction between cilia and flagella is mostly historical, as both organelles display a common architecture: cilia and flagella elongate their microtubules from a basal body forming a cylindrical structure termed the axoneme, composed of 9 doublets of microtubules. Most motile cilia exhibit a 9+2 structure, in which the axoneme surrounds a central pair of single microtubules. There are a few exceptions however, most strikingly the motile 9+0 cilia of the embryonic node (Nonaka et al., 1998) and the atypical spermatozoa of insects (Mencarelli et al., 2008). Various microtubule-associated appendages are involved in ciliary beating. Some generate the force necessary for motility such as outer dynein arms and inner dynein arms, while others regulate motor activity such as radial spokes and central pair projections. On the contrary, primary cilia have a $9+0$ axoneme, lack dynein arms and do not appear to be motile. Defects in flagellum assembly or function have been linked to an increasing number of genetic diseases collectively termed ciliopathies, such as for example primary ciliary dyskinesia, polycystic kidney disease, retinitis pigmentosa or the Bardet-Biedl syndrome (Huber and Cormier-Daire, 2012; Reiter and Leroux, 2017).

Cilia and flagella are complex organelles, composed of over 500 proteins (Pazour et al., 2005; Smith et al., 2005; Broadhead et al., 2006; Oberholzer et al., 2011; Subota et al., 2014), that must be incorporated into the flagellar axoneme, membrane or matrix. Cilia and flagella constitute a distinct compartment, as their content is separated from the rest of the cytoplasm by a barrier, or ciliary gate, positioned between the transition zone and the plasma membrane (Reiter et al., 2012). Since the cilium lacks ribosomes, its proteins must be synthesised in the cytoplasm prior to entry and incorporation into the organelle. This raises 
the issue of protein targeting and incorporation to the organelle during both construction and maintenance.

Intraflagellar transport (IFT) is a key process involved in flagellar construction. First identified in the green alga Chlamydomonas reinhardtii (Kozminski et al., 1993), it refers to the bidirectional transport of protein complexes along the axoneme, from the basal body to the distal tip of the axoneme and vice-versa, powered by the action of kinesin motors in the anterograde direction and dynein motor in the retrograde direction (Ishikawa and Marshall, 2011). IFT has since been shown to be conserved and essential for the assembly of almost all eukaryotic flagella (Hao and Scholey, 2009), and inhibition of IFT prevents flagellum assembly in most organisms studied so far (Kozminski et al., 1995; Nonaka et al., 1998; Brown et al., 1999; Han et al., 2003; Kohl et al., 2003). The canonical model for flagellum assembly proposes that flagellar components are transported by IFT to the distal tip of the flagellum, which is the site of construction of the organelle (Craft et al., 2015). Yet so far distal incorporation during flagellum construction has been formally demonstrated for relatively few structural proteins: alpha tubulin and the radial spoke protein RSP3 in Chlamydomonas (Johnson and Rosenbaum, 1992), and the PFR2 and KMP11 proteins in Trypanosoma brucei (Bastin et al., 1999a) (Zhou et al., 2015). In Chlamydomonas, analysis of dikaryons between wild-type and strains with various defects in structural elements, or with a tagged version of a given protein, revealed various profiles: addition at the distal end for tubulin, RSP3 (Johnson and Rosenbaum, 1992), the inner dynein arm subunit p28 (Piperno et al., 1996), the central pair protein PF6 (Lechtreck et al., 2013) or the dynein regulatory complex (DRC) subunit 4 (Bower et al., 2013). By contrast, proximal incorporation was observed for the docking complex of the outer dynein arm (Owa et al., 2014), and intercalation of the IC69 component was reported the outer dynein arm (Piperno et al., 1996) . Lateral diffusion has also been reported for the membrane protein Smoothened (Milenkovic et 
al., 2009). More recently proximal incorporation was demonstrated for the components of the associated flagellum attachment zone in Trypanosoma brucei (Zhou et al., 2015)(Sunter et al., 2015). The mode of incorporation could depend on the type of protein and on its final location.

In order to investigate the site of incorporation of various flagellar proteins and their dynamics, we turned to the protist $T$. brucei, the etiological agent of sleeping sickness in Africa that is a very amenable model for studying cilium biology (Vincensini et al., 2011). It possesses a single flagellum that remains present throughout the cell cycle and is composed of a typical 9+2 axoneme with central pair, dynein arms and radial spokes (Langousis and Hill, 2014), which is flanked by a lattice-like structure called the ParaFlagellar Rod (PFR)(Portman and Gull, 2010). The trypanosome assembles its new flagellum whilst maintaining the existing one, offering the opportunity to compare in the same cell an elongating flagellum with a flagellum undergoing maintenance. Moreover, the axoneme contains specific subdomains identified with unique proteins such as FLAM6 (restricted to the proximal part) and FLAM8 (only present at the distal tip)(Subota et al., 2014). This restricted protein localisation has also been reported in other eukaryotes including humans (Fliegauf et al., 2005; Yagi et al., 2009). Since multiple reverse genetics and imaging tools are available, trypanosomes are perfectly suited to study protein incorporation and turnover (Julkowska and Bastin, 2009; Oberholzer et al., 2009).

Here, we investigated the mode of addition to the growing flagellum of three proteins belonging to distinct elements of the axoneme (radial spokes, central pair, outer dynein arms) and of a membrane protein, using two experimental setups based either on rapid inducible expression of epitope-tagged versions of flagellar proteins, or on photobleaching experiments. The results reveal the existence of different modes of incorporation in both the old and the 
new flagellum, and highlight slow or no dynamics for structural proteins compared to the membrane protein that was studied.

\section{Results}

\section{Assembly of radial spoke and central pair proteins}

Radial spoke protein 3 (RSP3) incorporation into the growing flagellum of Chlamydomonas has been unambiguously shown to be distal (Johnson and Rosenbaum, 1992). Hence, we decided to first focus on this well-characterised protein to confirm that its mode of incorporation is indeed conserved in T. brucei. RSP3 is conserved in trypanosomes and its knockdown leads to the absence of radial spokes accompanied by a pronounced motility phenotype (Ralston et al., 2006). Flagellar incorporation of RSP3 was investigated using a strain expressing a Ty1 tagged version of the RSP3 protein (N-terminal tagging) under the control of a tetracycline inducible trypanosome promoter. The plasmid was transfected in a cell line constitutively expressing the tet-repressor, so that the promoter is silent under normal culture conditions, but can be rapidly activated upon addition of tetracycline (Wirtz and Clayton, 1995; Bastin et al., 1999a; Sunter et al., 2015).

This system allows the visualisation of recently synthesised proteins and the monitoring of their location during flagellum construction. Since the old flagellum is maintained while the new one is assembled, it is possible to monitor the fate of newly synthesised proteins within both flagella, and thus assess protein turnover in the mature flagellum. The strain displayed normal growth rate, unaltered by the addition of tetracycline, showing that the epitope-tagged protein is not toxic (data not shown). Western blotting with the Ty1 epitope tag specific monoclonal antibody BB2 was used to assess the incorporation of the tagged protein to the axoneme. Samples were treated with $1 \%$ NP40 to separate a 
cytoskeleton and a soluble fraction (Robinson et al., 1991). In non-induced cells, the level of TY1::RSP3 was low but detectable, indicating a slight leakiness of the system as previously reported (Wirtz and Clayton, 1995). Cells were induced for one hour by addition of tetracycline, leading to an increase in the amount of tagged protein (Figure 1A). The TY1::RSP3 protein fractionates in the cytoskeletal fraction (lanes C), confirming its incorporation to the axoneme. As observed for other axoneme or PFR proteins (Bastin et al., 1998; Baron et al., 2007; Kabututu et al., 2010; Ralston et al., 2011), the soluble pool turned out to be either absent or below detection level. However, these cells are not synchronised and the existence of a soluble pool during a brief phase of the cell cycle cannot be formally excluded. These results validate the strain as inducible, with expression kinetics compatible with flagellar assembly that takes 4-5 hours in cultured trypanosomes (Sherwin and Gull, 1989; Bastin et al., 1999a). In our experimental setup, the expression of the TY1::RSP3 tagged protein can be induced in just an hour, implying that its localisation provides a marker of recently synthesised proteins. As the pool of soluble flagellar proteins is low, newly synthesised proteins should also represent recently assembled material.

In order to determine the site of incorporation of RSP3, cells were induced with tetracycline, and TY1::RSP3 localisation was monitored over time by IFA. Cells were treated with $1 \%$ Nonidet prior to methanol fixation, in order to solubilise the cytoplasm and nonincorporated material. The remaining cytoskeletons were double labelled with BB2 to visualize the newly incorporated tagged RSP3, and with mAb25, an axoneme marker (Pradel et al., 2006). Observations were focused on bi-flagellated cells, in which the new assembling flagellum can be distinguished from the old flagellum based on its posterior position and its shorter length (Figure 1B) (Sherwin and Gull, 1989). Short incubations with tetracycline (1h) lead to expression of the fusion protein that was mostly localised at the distal tip of the new flagellum (Figure 1C, top panel). This profile was reproduced after $1 \mathrm{~h} 30 \mathrm{~min}$ of growth in the 
presence of tetracycline, where quantification revealed that more than $95 \%$ of the cells exhibited this staining profile $(\mathrm{n}=53)$. The remaining cells possessed a short flagellum that was fully stained, presumably because its assembly was initiated during the induction period (Figure 1C left panel). The length of the labelled segment increased with the duration of growth in the presence of tetracycline, in agreement with flagellar elongation rate. The length of the new flagellum segment showing bright positive signal was measured to $3.9 \pm 0.3 \mu \mathrm{m}$ (induction for $1 \mathrm{~h}, \mathrm{n}=45), 4.6 \pm 0.3 \mu \mathrm{m}(1 \mathrm{~h} 30, \mathrm{n}=53)$ and $7.1 \pm 0.5 \mu \mathrm{m}(2 \mathrm{~h} 30, \mathrm{n}=35)$, in good agreement with an elongation rate of $3.6 \mu \mathrm{m}$ per hour (Bastin et al., 1999a). After 4h of growth in the presence of tetracycline, a large segment of the distal part of long flagella was stained (Figure 1C, bottom panels). In all cases, the signal is resistant to detergent, showing that the tagged protein is indeed incorporated in the axoneme. These data support the view that radial spoke proteins are added to the distal end of the growing flagellum in trypanosomes.

We next wondered whether a protein located within a different sub-region of the axoneme could undergo a different mode of incorporation. We therefore investigated PF16, a well-characterised protein of the central pair (Smith and Lefebvre, 1996; Sapiro et al., 2002; Branche et al., 2006; Ralston et al., 2006), whose central position is more distant from the microtubule doublets that carry IFT particles. The same approach was used to generate an inducible cell line expressing Ty1-tagged PF16 upon addition of tetracycline. The strain displayed normal growth rate, unaltered by the addition of tetracycline, suggesting that the tagged protein is not toxic (data not shown). Expression was first monitored by western blotting, using the Ty1 tag specific antibody BB2 (Figure 2A). In non-induced cells, a low amount of tagged PF16 is visible, but upon tetracycline addition, the level of the protein increases slowly and reaches much higher levels after the cells have been induced for over 48h. The protein is restricted to the cytoskeletal fraction and is not detected within the soluble 
fraction. These results validate the strain as inducible, with kinetics acceptable for investigation of protein localisation during flagellum construction.

In order to determine the site of incorporation of PF16, cells were induced with tetracycline for 1 to $3 \mathrm{~h}$, and Ty1::PF16 expression was monitored over time by IFA. Cells were treated with $1 \%$ Nonidet prior to methanol fixation, in order to confirm axonemal incorporation. Slides were analysed by IFA double labelled with BB2, to visualize the newly incorporated tagged PF16, and with the flagellar marker mAb25. Short incubations with tetracycline ( $1 \mathrm{~h}$ and $1 \mathrm{~h} 30 \mathrm{~min}$ ) did not lead to detectable expression of Ty1::PF16 (data not shown). After $2 \mathrm{~h} 30 \mathrm{~min}$ of induction, the fusion protein was detected at the distal tip of the new flagellum (Figure 2B). Moreover, this signal was resistant to detergent, showing that the protein is indeed incorporated in the axoneme. A weak signal could be detected in the old flagellum, corresponding to a low level of expression of Ty1::PF16 due to a slight leakiness of the inducible system (as also observed on the western blot), but it did not show a particular polarity (Figure 2B). These results demonstrate that central pair proteins are added to the distal end of the elongating flagella, as observed for radial spokes.

\section{Photobleaching analysis of a dynein arm component during flagellum construction and}

\section{maintenance}

We next investigated the incorporation of a component of the outer dynein arm, the dynein intermediate chain 1 (DNAI1), which is located in the periphery of the axoneme (Branche et al., 2006). We developed a cell line where DNAI1 is endogenously tagged with GFP for photobleaching analysis. Western blotting analysis was performed with an anti-GFP, or with a mouse anti-DNAI1 polyclonal antibody (Duquesnoy et al., 2009) on total protein samples, cytoskeletal and detergent-soluble fractions (Figure 3A). The fusion protein was detected with both antibodies, whereas the untagged endogenous DNAI1 only reacted with the anti-DNAI1 
antibody. This revealed that the fluorescent version represents about half of the endogenous one. Both proteins showed the same distribution profile with more material in the cytoskeletal fraction than in the soluble fraction (Figure 3A) as previously observed (Duquesnoy et al., 2009). Live video-microscopy showed that, as expected, the protein is constitutively expressed and localises to both old and new flagella (Figure 3B).

DNAI1 flagellar incorporation and dynamics were investigated using fluorescence recovery after photobleaching (FRAP). In bi-flagellated cells, the fluorescent signal was bleached in both flagella and fluorescence recovery was monitored simultaneously in the mature and in the elongating flagellum. We decided to photobleach the new flagellum in its entirety but only half of the old flagellum, hence leaving a positive signal to control for bleaching due to laser exposure and to facilitate cell detection. Evolution of the fluorescent signal was monitored for up to $2 \mathrm{~h}$. In all cases, no recovery was detected in the old flagellum (Figure 3B). By contrast, a fluorescent signal became detectable in the new flagellum from half an hour after photobleaching (Figure 3B, time 33 minutes). It was mostly present towards the distal tip, but some signal was also detected towards the proximal part (Figure 3B). A clear gradient was visible at later time points with stronger signal at the distal tip (Figure 3B). The new flagellum showed signs of conspicuous elongation during the course of the experiment and so the bright signal corresponds to the incorporation of new GFP::DNAI proteins on the growing axoneme. Presence of a positive (albeit less bright) signal in the adjacent proximal region that was already assembled before the bleach could reflect either turnover of material that had already been incorporated or completion of the assembly by an intercalation process. This suggests a clear difference in protein dynamic between the new flagellum and the old flagellum where no turnover is observed.

However, turnover could still occur at the distal end of the mature flagellum, that is known to be highly dynamic in some species such as Chlamydomonas (Marshall and 
Rosenbaum, 2001), or C. elegans (Hao et al., 2011). To investigate whether dynein turnover may occur in the distal portion of the flagellum of $T$. brucei, the fluorescent signal was bleached at the distal tip of the flagellum in cells expressing GFP::DNAI1, and fluorescence recovery was monitored. No recovery could be detected at the distal tip (Supplementary Figure 1). This experiment was reproduced using a cell line expressing the dynein heavy chain ODA-B fused to mNeonGreen following in situ tagging at the 5' end of the gene (Shen et al., 2001). The distal end of the mature flagellum was bleached (Figure 4A) and recovery was monitored over 45min (Figure 4B-G). Again, no recovery was observed. These results demonstrate that both the heavy and the intermediate dynein chains undergo little or no turn over at the distal tip of the flagellum, supporting the view that, once assembled, mature trypanosome flagella do not modify their length (Ooi and Bastin, 2013).

Overall these results show that the structural proteins of the axoneme studied so far mostly follow a distal pattern of incorporation, with a slight variation for the outer dynein arm DNAI1 protein that appears to also undergo intercalation or turnover at the proximal part of the elongating flagellum.

\section{The flagellum membrane-associated protein AK3 shows fast and non-polarised incorporation in growing or mature flagella}

A recent study indicated that flagellar proteins display diverse dynamic behaviours depending on their flagellar localisation (Subota et al., 2014). To investigate this phenomenon, we turned towards a novel flagellum membrane protein called arginine kinase 3 (AK3) (Oberholzer et al., 2011; Voncken et al., 2013; Subota et al., 2014; Ooi et al., 2015). In contrast to structural axonemal proteins, detergent extraction localised AK3 exclusively to the soluble fraction and IFA data unambiguously revealed that AK3 co-localises with the flagellum membrane and wraps around all flagellar structural elements such as the axoneme and the PFR (Subota et al., 
2014). The staining is distinct from that observed for intraflagellar proteins and is sensitive to detergent treatment (Subota et al., 2014). This staining profile is very similar to that observed for the flagellar membrane proteins calflagins (Maric et al., 2011). The T. brucei genome contains 3 genes encoding closely related proteins for arginine kinase that differ in their N- or C-termini sequences (Miranda et al., 2009). These sequences are responsible for differential locations: AK1 is in the cytosol, AK2 is in the glycosomes and AK3 is in the flagellum membrane (Voncken et al., 2013; Ooi et al., 2015). RNAi silencing leads to rapid disappearance of AK3 in both old and new flagella in less than $4 \mathrm{~h}$, corresponding to one third of the duration of the cell cycle. This disappearance did not show a particular polarity in IFA experiments, suggesting that AK3 might move rapidly in the flagellum membrane (Subota et al., 2014).

An inducible strain expressing AK3::Ty1 under the control of the tetracycline repressor was generated in an ak3-/- knockout strain: the tagged protein being expressed in a negative genetic background, there is no competition with the endogenous AK3 protein. Tetracycline-inducible expression was confirmed by western blotting, using the anti-Ty1 tag BB2 antibody and the anti-AK polyclonal antibody (Figure 5A). In non-induced cells, no AK3::Ty1 is detectable, neither by BB2 nor by the anti-AK antiserum. Upon tetracycline addition, the level of the tagged protein increases rapidly as confirmed by detection with both $\mathrm{BB} 2$ and the anti-AK antiserum, which detects both AK1/AK2 proteins (these two co-migrate at $\sim 40 \mathrm{kDa}$ ) and the tagged AK3 protein. These results validate the strain as inducible, with fast kinetics compatible with flagellar dynamics.

Induction experiments were then analysed by IFA with either the BB2 or the anti-AK antibody. Upon 30 minutes of induction, both flagella were equally labelled, with no significant difference in intensity between them (Figure 5B). This was consistently observed no matter the length of the new flagellum (Figure 5B). Neither a particular polarity nor a 
gradient could be observed. These results were reproduced using a cell line expressing the Ty1-tagged version of AK3 in a background where both $A K 3$ endogenous alleles were still present, showing that these distribution profiles are not explained by the fact that $\mathrm{AK} 3$ is absent from the mature flagella before induction (data not shown). These results illustrate fast turn over in both flagella and do not exclude the possibility of rapid exchange between the two flagella could also be considered.

IFT has long been postulated to transport flagellar components within the organelle and recent work has shown that several proteins rely on IFT to be maintained at their correct location in the flagellum (Fort et al., 2016). The possible contribution of IFT to the flagellar incorporation of AK3 was investigated using two tetracycline-inducible RNAi strains: $I F T 88^{R N A i}$ and $I F T 140^{R N A i}$ in which respectively anterograde and retrograde transport are inhibited upon knockdown (Kohl et al., 2003; Absalon et al., 2008). Cells were stained with the anti-AK antibody and an antibody against the Flagellum Transition Zone Component FTZC, as marker of the base of the flagellum (Bringaud et al., 2000). In non-induced conditions, the signal covered the flagellum membrane as expected (Figure 6A). Over the course of RNAi silencing, the amount of IFT is reduced and cells assemble shorter and shorter flagella. However, mature flagella that were assembled prior to initiation of RNAi remain present (Figure 6B,C), even though IFT is absent (IFT88 knockdown), or arrested (IFT140 knockdown)(Fort et al., 2016). This system thus allows the investigation of the contribution of IFT to flagellar targeting of AK and its distribution within the flagellum. The AK signal was retained in all flagella of induced $I F T 88^{R N A i}$ and $I F T 140^{R N A i}$ cells (arrows on Figures 6B,C), no matter their length, indicating that flagellar targeting of $\mathrm{AK}$ is probably independent of IFT. In cells lacking flagella, the signal for AK was significantly increased in the cytoplasm (Figure 6B,C). This could correspond to AK3 still being produced and accumulating there in the absence of flagellum, or it could represent an increase in the amount 
of AK1 and AK2 that have been located to the cytoplasm and to glycosomes respectively (Voncken et al., 2013).

\section{Discussion}

This study revealed two distinct protein behaviours during flagellum construction and maintenance. Structural proteins are mostly added at the distal end of the elongating organelle with little (if any) turnover in mature flagella, whereas membrane proteins do not show a specific polarity during incorporation and exhibit a rapid turnover. Components of the radial spokes, the central pair and the dynein arms (RSP3, PF16 and DNAI1) are mainly assembled at the distal end of the growing T. brucei axoneme. This is in agreement with the established observation for PFR2, a major component of the PFR (Bastin et al., 1999a) and supports the view that this mode of incorporation is conserved for components of flagellar skeletal structures among eukaryotic species (Rosenbaum et al., 1969; Johnson and Rosenbaum, 1992; Lechtreck et al., 2013). The approaches used here have the advantage of visualising addition of new subunits in elongating flagella, rather than in the dikaryon experiments where flagella are already assembled.

Distal assembly is compatible with IFT transport of components of the axoneme and the PFR. FRAP analyses in Chlamydomonas have convincingly demonstrated transport of alpha-tubulin, dynein regulatory complex components and PF16 in growing and mature flagella (Wren et al., 2013; Craft et al., 2015). So far, IFT movement of axoneme or PFR proteins has not been shown directly in trypanosomes. Epitope- or YFP-tagged alpha-tubulin fails to incorporate into flagellar microtubules, hence hampering direct analysis (Bastin et al., 1996; Sheriff et al., 2014). Here, monitoring of the elongating flagellum after photobleaching of the GFP::DNAI1 signal failed to reveal IFT-type movement. This could be explained by 
technical reasons if the amount of DNAI1 per IFT train was too low to be detected, or if the association of the cargo to the IFT train complex interfered with the ability of GFP to fluoresce.

Alternatively, outer dynein arms might not rely on IFT for transport in the trypanosome flagellum. In Chlamydomonas, analysis of dikaryons between dynein arm and IFT mutants suggested that inner dynein arms, but not outer dynein arms, require a functional IFT kinesin for correct incorporation (Piperno et al., 1996). However, further work has shown that outer dynein arm addition was dependent on IFT46 (Hou et al., 2007) and the IFTassociated adaptor protein ODA16 (Ahmed et al., 2008). These data support a contribution of IFT, but visualisation of the way ODA components move within the flagellum will be needed to formally prove whether they are transported by IFT or by other means. For example, the distal protein EB1 is added at the distal end of axonemal microtubules independently of IFT (Harris et al., 2016).

Transport of flagellar precursors was suggested in the $P F R 2^{R N A i}$ mutant that fails to assemble a normal PFR (Bastin et al., 1998) in which other PFR proteins are found at the distal end of the flagellum where they accumulate over the course of organelle assembly. This material is not incorporated in any structure and is lost after cell division (Bastin et al., 1999b). However, formal evidence of IFT-like movement of PFR precursors is still lacking and the possibility of diffusion (Ye et al., 2013), or the use of other types of motor systems (Demonchy et al., 2009) cannot be ruled out.

Although distal incorporation of structural axoneme proteins was the major trend in both series of experiments (inducible expression of tagged proteins and FRAP experiments), some signal was detected towards the proximal part of the assembling flagellum for DNAI1, often visible as a gradient starting from the distal region. One could imagine that the majority of the material destined for incorporation is delivered at the distal tip to be associated to 
"naked", recently elongated microtubule segments but that some material is released from the IFT train prior to reaching the tip of the flagellum. These proteins would serve for completion of the assembly or for turnover. This hypothesis is supported by the relative structural disorganisation found in the terminal portion of the new flagellum of T. brucei perhaps because assembly of all subcomponents is not complete (Hoog et al., 2014). In this context, it should be pointed out that in Chlamydomonas, DRC4 can dissociate from IFT trains at various sites along the flagellum and not always at the distal tip (Wren et al., 2013).

The situation turned out to be very different for the membrane-associated flagellar protein AK3, where no polarity could be detected during assembly of the flagellum. AK3 is found all along the flagellum membrane without any specific association to a defined substructure (Subota et al., 2014). It is likely associated to the membrane by prenylation, as suggested by the presence of a typical flagellum-targeting signal at its amino-terminus (Ooi et al., 2015) similar to that found in calflagins (Godsel and Engman, 1999; Maric et al., 2011). These proteins presumably associate first to the cell body membrane and then to the flagellum membrane (Emmer et al., 2009). Although direct trafficking studies of calflagins have not been reported, these proteins likely reach the flagellum by its base and then could either diffuse within the organelle, or be associated or transported by systems yet to discover. In cultured mammalian cells, two trans-membrane proteins (the somatostatin receptor 3 and Smoothened, the Hedgehog transducer) appeared to diffuse freely within the primary cilium (Ye et al., 2013). RNAi showed that all the AK3 flagellar protein pool is turned over in less than $4 \mathrm{~h}$ (Subota et al., 2014), which is less than half of the trypanosome cell cycle. This shows that the old flagellum is dynamic with regards to rapid replacement of at least certain membrane proteins. AK3 is a phosphagen shuttle and has been proposed to contribute to flagellum motility (Voncken et al., 2013; Ooi et al., 2015). Since both flagella appear equally motile, the requirements for AK3 could be similar. 
During half of their cell cycle, trypanosomes possess two basal bodies and two flagella, one undergoing construction and one that is already fully assembled (Sherwin and Gull, 1989). As discussed above, newly synthesised subunits for structural proteins are mostly targeted to the new flagellum with weak amounts reaching the old flagellum. In mature flagella of Chlamydomonas, the degree of protein exchange turned out to be highly variable: some axonemal proteins hardly showed any turnover, whereas others were totally replaced during the life of the flagellum (Song and Dentler, 2001). Protein exchange requires the existence of a soluble pool of material that could not be detected in the case of PFR (Bastin et al., 1998) or DRC proteins (Kabututu et al., 2010; Ralston et al., 2011), but exists in significant amounts for the dynein component LC1 (Ralston et al., 2011), and to a lesser extent for the inner dynein arm component 5 (Wei et al., 2014) and for DNAI1 (Duquesnoy et al., 2009). A large soluble pool might not be necessary providing that mRNA is available and that translation produces protein that is immediately used for turnover. Analysis of mRNA content at different cell cycle stages from trypanosomes that had been synchronised by elutriation revealed that many flagellar genes are up-regulated during flagellum synthesis (Archer et al., 2011). Moreover, the timing of the expression peaks reflects that of flagellum construction: transcripts for basal body and IFT proteins emerge first, followed by those for axonemal proteins and ultimately those for PFR proteins (Morga and Bastin, 2013).

Finally, the absence of turnover of both the heavy and the intermediate dynein chains supports the view that the length of the mature flagellum in trypanosomes is fixed (Ooi and Bastin, 2013). This finding explains that absence or arrest of IFT in mature flagella had no effect on their length, contrarily to the growing flagellum (Fort et al., 2016). This means that the control of flagellum length is different compared to what has been described for Chlamydomonas, where flagellar length is regulated by a dynamic balance of assembly 
(relying on IFT for continuous delivery of tubulin) and disassembly at the distal end (Marshall and Rosenbaum, 2001). 


\section{Materials and methods}

Trypanosome cell line and culture — For inducible expression, procyclic trypanosome cell lines were generated from the PTH cell line, a derivative of strain 427 that constitutively expresses the tet-repressor (Bastin et al., 1999a). $I 788^{R N A i}$ (Kohl et al., 2003) and IFT140 ${ }^{R N A i}$ (Absalon et al., 2008) strain have been described previously. They were cultured in SDM-79 (Brun and Schonenberger, 1979) supplemented with hemin and $10 \%$ fetal bovine serum, with the exception of the inducible cell line expressing the AK3::Ty1 protein that was grown in SDM79 supplemented with $20 \mathrm{mM}$ glycerol (Ooi et al., 2015). The AnTat1.1 strain was used for expression of mNeonGreen::DHCODAB. Long primer PCR transfections were performed in bloodstream form parasites cultured in HMI-11 medium prior to differentiation into procyclic stage parasites. Bloodstream pleomorphic parasites were differentiated by addition of $5 \mu \mathrm{M} 8$-pCPT-2-O-methyl-5-AMP to $10^{5}$ parasites per $\mathrm{ml}$ in $10 \mathrm{ml}$ of HMI-11. Parasites were cultured for 48 hours to induce stumpy formation followed by transfer to SDM-79 medium supplemented with $20 \mathrm{mM}$ glycerol and $6 \mathrm{mM}$ cis-aconitate at $10^{6}$ parasites per $\mathrm{ml}$ and transferred to a $27^{\circ} \mathrm{C}$ incubator for $72 \mathrm{~h}$, after which time cells were cultured in SDM-79 supplemented with $20 \mathrm{mM}$ glycerol. Cell numbers in culture was determined using the Z2 cell counter (Beckman Coulter).

Expression of Ty1 and fluorescent fusion proteins - Expression of Ty1-tagged flagellar proteins was achieved with the pHD430 plasmid that contains the full gene sequence fused to the Ty1 tag in 5' (RSP3) or 3' (PF16) positions, under the control of the tetracycline-inducible EP promoter (Bastin et al., 1999a). All the sequence fragments were chemically synthesized by GeneCust Europe (Dudelange, Luxembourg) and sub-cloned into the pHD430 vector. For the generation of cell lines expressing these Ty1 fusion proteins, linearized pHD430 vectors were nucleofected into PTH cells that express the tetracycline-repressor by targeting the 
inverted spacer of the ribosomal DNA that is supposed to be silent (Wirtz and Clayton, 1995). For endogenous tagging of DNAI1, the first $400 \mathrm{bp}$ of the gene were cloned in the pPCPFRGFPDHC1b vector (Blisnick et al., 2014) and integrated in the genome following linearization within the DNAII sequence. For in situ tagging of DHC-ODAB (Tb927.11.3250), primers matching the p2675mNeonGreenIFT81 plasmid were designed with $80 \mathrm{bp}$ extensions covering the last $80 \mathrm{bp}$ of the 5'UTR sequence (CGT GTC CGT AGG TGG AAC GAT TAA GCA ACG AGA AGA GGA GTT ACG TAA ATC AAA CAA GCA AAC TAA GGA AAG GAA CCC CGC CTA AAG TCG AGG AGG TTG A) and the first 80 bp of the DHC-ODAB coding sequence ((TTT AAC CCG GTA ATG ATG CGC TGC TCA AGC CAC TGA ACA CGC CTA TCG ACG GGT GCC TCT TCC TTG TCG CCC TTC GCC ATG TCA AGT GGG TCC TGG TTA G), hence amplifying the puromycin drug resistance cassette, the splicing sequence, a Ty-1 tag and the mNeonGreen sequence. Transfections were carried out using Nucleofector ${ }^{\circledR}$ technology (Lonza, Italy)(Burkard et al., 2007). Transgenic cell lines were selected in medium supplemented with phleomycin (2.5 $\mu \mathrm{g} / \mathrm{ml})$ or puromycin $(1 \mu \mathrm{g} / \mathrm{ml})$ where appropriate.

\section{Tetracycline induction time course}

Tetracycline induction was carried out at a concentration of $1 \mu \mathrm{g} / \mathrm{ml}$. Cells were grown in culture to $\sim 10^{7}$ cells $/ \mathrm{ml}$ in SDM79 or SDMG medium prior to commencement of the experiment. Non-induced cells were split into separate flasks corresponding to the number of time points and induction was initiated in a staggered manner. Upon addition of tetracycline (Sigma) to the flask for time 0, cells were harvested for western blotting or immunofluorescence assays. 
Immunoblot analysis - Samples were boiled in Laemmli buffer (2x stock: 0.5 M Tris pH 6.8 containing 20\% Glycerol, 4\% DTT, 4\% SDS, Bromo-phenol blue) before SDS-PAGE separation, loading $40 \mu \mathrm{g}$ of total cell protein per lane. The Criterion system (Biorad) was used for electrophoresis. Proteins were transferred to PVDF membranes (Hybond-P from Amersham) in the Criterion blotter (Biorad) for 45 minutes at $100 \mathrm{~V}$ constant in TG buffer (10x stock: $0.25 \mathrm{mM}$ Tris $\mathrm{pH} 8.3,1.92 \mathrm{mM}$ glycine). The membrane was blocked overnight with $5 \%$ skimmed milk in PBS and incubated with primary antibodies diluted in $1 \%$ milk and $0.1 \%$ Tween 20 in PBS for $1 \mathrm{~h}$. Membrane washes were performed with $0.2 \%$ Tween20 in PBS. Species specific secondary antibodies coupled to HRP (GE Healthcare) were diluted $1 / 20,000$ in $1 \%$ milk and $0.1 \%$ Tween 20 in PBS and incubated with the membranes for $1 \mathrm{~h}$. Final detection was carried out by using an ECL kit according to manufacturer's instructions (Amersham) and exposure of Hyperfilm-ECL (Amersham). Antibodies against the endoplasmic reticulum component BiP (Bangs et al., 1993) or against the paraflagellar proteins (Kohl et al., 1999) were used as loading controls.

Indirect immunofluorescence assay (IFA)_Cultured parasites were washed twice in SDM79 medium without serum and spread on poly-L-lysine coated slides (Menzel-Gläser, Braunschweig) before fixation. For methanol fixation, parasites were air dried and fixed in methanol at $-20^{\circ} \mathrm{C}$ for 5 minutes followed by a rehydration step for 15 minutes in PBS. For PFA fixation, parasites were left to settle on slides, rinsed in PBS before being incubated for 30 minutes at room temperature with a 4\% PFA solution in PBS at $\mathrm{pH} 7$. After a permeabilization step with $0.1 \%$ Nonidet P-40 (Fluka) in PBS, samples were blocked for 1 hour with 1\% BSA in PBS. To extract the cytoskeleton and solubilise cytoplasmic contents, the cells were left to settle on poly-L-lysine coated slides for 10 minutes, rinsed in PBS and treated for 7 seconds with 1\% NP40 in PEM buffer (0.1 M PIPES pH 6.9, 2 mM EGTA, 
$1 \mathrm{mM} \mathrm{MgSO}_{4}$ ). After thorough washes, the samples were fixed in methanol before being processed.

For immunodetection, slides were incubated with the appropriate dilution of the first antibody in $0.1 \%$ BSA in PBS for 1 hour. MAb25 recognises the axonemal protein TbSAXO1 (Dacheux et al., 2012) and was used as a marker of the axoneme (Pradel et al., 2006) while BB2 served to detect the Ty1 tag (Bastin et al., 1996). After three 5 minute-washes, species and subclass-specific secondary antibodies coupled to the appropriate fluorochrome (Alexa 488, Cy3 or Cy5, Jackson ImmunoResearch) were diluted 1/400 in PBS containing $0.1 \%$ BSA and were applied for 1 hour. After washing as above, cells were stained with a $1 \mu \mathrm{g} / \mathrm{ml}$ solution of the DNA-dye DAPI (Roche) and mounted with the ProLong antifade reagent (Invitrogen). Slides were analysed with a DMR microscope (Leica) and images captured with a CoolSnap HQ camera (Roper Scientific). Image acquisition was controlled using ImageJ and images were taken with the threshold set at maximum. Subsequent normalization of signals was carried out by parallel manipulation of brightness and contrast against controls, and images were superimposed using Photoshop CS4.

\section{Fluorescent Recovery After Photobleaching (FRAP) analysis - The expression of} GFP::DNAI1 was first observed directly with a DMI4000 Leica microscope using a mercury bulb for excitation to verify correct protein expression and localisation. For FRAP analysis of cells expressing GDP::DNAI1, a Zeiss inverted microscope (Axiovert 200) equipped with an oil immersion objective (magnification x63 with a 1.4 numerical aperture) and a spinning disk confocal head (CSU22, Yokogawa) was used (Buisson et al., 2013). Images were acquired using Volocity software with an EMCCD camera (C-9100, Hamamatsu) operating in streaming mode. A sample was taken directly from the culture grown at 6 to $8 \times 10^{6}$ cells $/ \mathrm{mL}$ and trapped between slide and coverslip. The samples were kept at $27^{\circ} \mathrm{C}$ using a fast response 
mini-stage temperature controller. Time-lapse sequences were acquired to analyse GFP signal recovery after photobleaching. Movies were taken using a time lapse of 3 minutes. Exposure time was 0.8 second per frame (binning was $1 \mathrm{x} 1$ pixels). In the case of cells expressing mNeonGreen::DHC-ODAB, the same settings were used except that the microscope was equipped with a 100x objective (1.4 numerical aperture). In this case, 8 cells were identified per series and their position recorded before photobleaching. Sequences of 20 seconds were filmed for each with an exposure time of 0.1 second per frame. Time lapse varied between 5 and 13 minutes. 


\section{Acknowledgements}

We thank Derrick Robinson (Bordeaux) for providing the Mab25 antibody. We thank the Imagopole for providing access to their equipment and Jean-Yves Tinevez for training and valuable advice.

\section{Funding}

L.V. was supported by a Roux post-doctoral fellowship from the Institut Pasteur. E.B. is supported by fellowships from French National Ministry for Research and Technology (doctoral school CDV515) and from La Fondation pour la Recherche Médicale (FDT20170436836). S. H. is funded by Roux post-doctoral fellowship from the Institut Pasteur. This work is funded by ANR grants (11-BSV8-016 and 14-CE35-0009-01), by a French Government Investissement d'Avenir programme, Laboratoire d'Excellence "Integrative Biology of Emerging Infectious Diseases" (ANR-10-LABX-62-IBEID) and by La Fondation pour la Recherche Médicale (Equipe FRM DEQ20150734356). The funders had no role in the study design, data collection and analysis, decision to publish or preparation of the manuscript.

\section{Author contributions}

L.V. \& P.B. conceived and designed the experiments; L.V., T.B., E.B., S.H., C.P.O and C.G. performed the experiments; L.V. and P.B. wrote the manuscript. T.B. prepared the figures, all authors commented on the manuscript.

\section{Conflict of interest}

The authors have declared no conflict of interest. 


\section{References}

Absalon, S., Blisnick, T., Kohl, L., Toutirais, G., Dore, G., Julkowska, D., Tavenet, A., and Bastin, P. (2008). Intraflagellar transport and functional analysis of genes required for flagellum formation in trypanosomes. Mol Biol Cell 19, 929-944.

Ahmed, N.T., Gao, C., Lucker, B.F., Cole, D.G., and Mitchell, D.R. (2008). ODA16 aids axonemal outer row dynein assembly through an interaction with the intraflagellar transport machinery. The Journal of cell biology 183, 313-322.

Archer, S.K., Inchaustegui, D., Queiroz, R., and Clayton, C. (2011). The cell cycle regulated transcriptome of Trypanosoma brucei. PLoS One 6, e18425.

Bangs, J.D., Uyetake, L., Brickman, M.J., Balber, A.E., and Boothroyd, J.C. (1993).

Molecular cloning and cellular localization of a BiP homologue in Trypanosoma brucei.

Divergent ER retention signals in a lower eukaryote. Journal of cell science 105 ( Pt 4), 11011113.

Baron, D.M., Ralston, K.S., Kabututu, Z.P., and Hill, K.L. (2007). Functional genomics in Trypanosoma brucei identifies evolutionarily conserved components of motile flagella. Journal of cell science 120,478-491.

Bastin, P., Bagherzadeh, Z., Matthews, K.R., and Gull, K. (1996). A novel epitope tag system to study protein targeting and organelle biogenesis in Trypanosoma brucei. Mol Biochem Parasitol 77, 235-239.

Bastin, P., MacRae, T.H., Francis, S.B., Matthews, K.R., and Gull, K. (1999a). Flagellar morphogenesis: protein targeting and assembly in the paraflagellar rod of trypanosomes. Mol Cell Biol 19, 8191-8200. 
Bastin, P., Pullen, T.J., Sherwin, T., and Gull, K. (1999b). Protein transport and flagellum assembly dynamics revealed by analysis of the paralysed trypanosome mutant snl-1. Journal of cell science 112 ( Pt 21), 3769-3777.

Bastin, P., Sherwin, T., and Gull, K. (1998). Paraflagellar rod is vital for trypanosome motility. Nature 391, 548.

Blisnick, T., Buisson, J., Absalon, S., Marie, A., Cayet, N., and Bastin, P. (2014). The intraflagellar transport dynein complex of trypanosomes is made of a heterodimer of dynein heavy chains and of light and intermediate chains of distinct functions. Mol Biol Cell 25, 2620-2633.

Bower, R., Tritschler, D., Vanderwaal, K., Perrone, C.A., Mueller, J., Fox, L., Sale, W.S., and Porter, M.E. (2013). The N-DRC forms a conserved biochemical complex that maintains outer doublet alignment and limits microtubule sliding in motile axonemes. Mol Biol Cell 24, 1134-1152.

Branche, C., Kohl, L., Toutirais, G., Buisson, J., Cosson, J., and Bastin, P. (2006). Conserved and specific functions of axoneme components in trypanosome motility. Journal of cell science $119,3443-3455$.

Bringaud, F., Robinson, D.R., Barradeau, S., Biteau, N., Baltz, D., and Baltz, T. (2000). Characterization and disruption of a new Trypanosoma brucei repetitive flagellum protein, using double-stranded RNA inhibition. Mol Biochem Parasitol 111, 283-297.

Broadhead, R., Dawe, H.R., Farr, H., Griffiths, S., Hart, S.R., Portman, N., Shaw, M.K., Ginger, M.L., Gaskell, S.J., McKean, P.G., and Gull, K. (2006). Flagellar motility is required for the viability of the bloodstream trypanosome. Nature 440, 224-227.

Brown, J.M., Marsala, C., Kosoy, R., and Gaertig, J. (1999). Kinesin-II is preferentially targeted to assembling cilia and is required for ciliogenesis and normal cytokinesis in Tetrahymena. Mol Biol Cell 10,3081-3096. 
Brun, R., and Schonenberger. (1979). Cultivation and in vitro cloning or procyclic culture forms of Trypanosoma brucei in a semi-defined medium. Short communication. Acta tropica 36, 289-292.

Buisson, J., Chenouard, N., Lagache, T., Blisnick, T., Olivo-Marin, J.C., and Bastin, P. (2013). Intraflagellar transport proteins cycle between the flagellum and its base. Journal of cell science $126,327-338$.

Burkard, G., Fragoso, C.M., and Roditi, I. (2007). Highly efficient stable transformation of bloodstream forms of Trypanosoma brucei. Molecular and biochemical parasitology 153, 220-223.

Craft, J.M., Harris, J.A., Hyman, S., Kner, P., and Lechtreck, K.F. (2015). Tubulin transport by IFT is upregulated during ciliary growth by a cilium-autonomous mechanism. The Journal of cell biology 208, 223-237.

Dacheux, D., Landrein, N., Thonnus, M., Gilbert, G., Sahin, A., Wodrich, H., Robinson, D.R., and Bonhivers, M. (2012). A MAP6-related protein is present in protozoa and is involved in flagellum motility. PloS one 7, e31344.

Demonchy, R., Blisnick, T., Deprez, C., Toutirais, G., Loussert, C., Marande, W., Grellier, P., Bastin, P., and Kohl, L. (2009). Kinesin 9 family members perform separate functions in the trypanosome flagellum. J Cell Biol 187, 615-622.

Duquesnoy, P., Escudier, E., Vincensini, L., Freshour, J., Bridoux, A.M., Coste, A., Deschildre, A., de Blic, J., Legendre, M., Montantin, G., Tenreiro, H., Vojtek, A.M., Loussert, C., Clement, A., Escalier, D., Bastin, P., Mitchell, D.R., and Amselem, S. (2009). Loss-of-function mutations in the human ortholog of Chlamydomonas reinhardtii ODA7 disrupt dynein arm assembly and cause primary ciliary dyskinesia. Am J Hum Genet 85, 890896. 
Emmer, B.T., Souther, C., Toriello, K.M., Olson, C.L., Epting, C.L., and Engman, D.M. (2009). Identification of a palmitoyl acyltransferase required for protein sorting to the flagellar membrane. Journal of cell science 122, 867-874.

Fliegauf, M., Olbrich, H., Horvath, J., Wildhaber, J.H., Zariwala, M.A., Kennedy, M., Knowles, M.R., and Omran, H. (2005). Mislocalization of DNAH5 and DNAH9 in respiratory cells from patients with primary ciliary dyskinesia. American journal of respiratory and critical care medicine $171,1343-1349$.

Fort, C., Bonnefoy, S., Kohl, L., and Bastin, P. (2016). Intraflagellar transport is required for the maintenance of the trypanosome flagellum composition but not its length. Journal of cell science $129,3026-3041$.

Godsel, L.M., and Engman, D.M. (1999). Flagellar protein localization mediated by a calcium-myristoyl/palmitoyl switch mechanism. EMBO J. 18, 2057-2065.

Han, Y.G., Kwok, B.H., and Kernan, M.J. (2003). Intraflagellar transport is required in Drosophila to differentiate sensory cilia but not sperm. Curr Biol 13, 1679-1686.

Hao, L., and Scholey, J.M. (2009). Intraflagellar transport at a glance. Journal of cell science $122,889-892$.

Hao, L., Thein, M., Brust-Mascher, I., Civelekoglu-Scholey, G., Lu, Y., Acar, S., Prevo, B., Shaham, S., and Scholey, J.M. (2011). Intraflagellar transport delivers tubulin isotypes to sensory cilium middle and distal segments. Nat Cell Biol 13, 790-798.

Harris, J.A., Liu, Y., Yang, P., Kner, P., and Lechtreck, K.F. (2016). Single-particle imaging reveals intraflagellar transport-independent transport and accumulation of EB1 in Chlamydomonas flagella. Mol Biol Cell 27, 295-307.

Hoog, J.L., Lacomble, S., O'Toole, E.T., Hoenger, A., McIntosh, J.R., and Gull, K. (2014). Modes of flagellar assembly in Chlamydomonas reinhardtii and Trypanosoma brucei. eLife 3, $\mathrm{e} 01479$. 
Hou, Y., Qin, H., Follit, J.A., Pazour, G.J., Rosenbaum, J.L., and Witman, G.B. (2007).

Functional analysis of an individual IFT protein: IFT46 is required for transport of outer dynein arms into flagella. The Journal of cell biology 176, 653-665.

Huber, C., and Cormier-Daire, V. (2012). Ciliary disorder of the skeleton. American journal of medical genetics. Part C, Seminars in medical genetics $160 C, 165-174$.

Ishikawa, H., and Marshall, W.F. (2011). Ciliogenesis: building the cell's antenna. Nat Rev Mol Cell Biol 12, 222-234.

Johnson, K.A., and Rosenbaum, J.L. (1992). Polarity of flagellar assembly in

Chlamydomonas. The Journal of cell biology 119, 1605-1611.

Julkowska, D., and Bastin, P. (2009). Tools for analyzing intraflagellar transport in trypanosomes. Methods Cell Biol 93, 59-80.

Kabututu, Z.P., Thayer, M., Melehani, J.H., and Hill, K.L. (2010). CMF70 is a subunit of the dynein regulatory complex. Journal of cell science $123,3587-3595$.

Kohl, L., Robinson, D., and Bastin, P. (2003). Novel roles for the flagellum in cell morphogenesis and cytokinesis of trypanosomes. Embo J 22, 5336-5346.

Kohl, L., Sherwin, T., and Gull, K. (1999). Assembly of the paraflagellar rod and the flagellum attachment zone complex during the Trypanosoma brucei cell cycle. J Eukaryot Microbiol 46, 105-109.

Kozminski, K.G., Beech, P.L., and Rosenbaum, J.L. (1995). The Chlamydomonas kinesinlike protein FLA10 is involved in motility associated with the flagellar membrane. The Journal of cell biology $131,1517-1527$.

Kozminski, K.G., Johnson, K.A., Forscher, P., and Rosenbaum, J.L. (1993). A motility in the eukaryotic flagellum unrelated to flagellar beating. Proc Natl Acad Sci U S A 90, 5519-5523. Langousis, G., and Hill, K.L. (2014). Motility and more: the flagellum of Trypanosoma brucei. Nature reviews. Microbiology 12, 505-518. 
Lechtreck, K.F., Gould, T.J., and Witman, G.B. (2013). Flagellar central pair assembly in Chlamydomonas reinhardtii. Cilia 2, 15.

Maric, D., McGwire, B.S., Buchanan, K.T., Olson, C.L., Emmer, B.T., Epting, C.L., and Engman, D.M. (2011). Molecular determinants of ciliary membrane localization of Trypanosoma cruzi flagellar calcium-binding protein. The Journal of biological chemistry 286, 33109-33117.

Marshall, W.F., and Rosenbaum, J.L. (2001). Intraflagellar transport balances continuous turnover of outer doublet microtubules: implications for flagellar length control. The Journal of cell biology $155,405-414$.

Mencarelli, C., Lupetti, P., and Dallai, R. (2008). New insights into the cell biology of insect axonemes. Int Rev Cell Mol Biol 268, 95-145.

Milenkovic, L., Scott, M.P., and Rohatgi, R. (2009). Lateral transport of Smoothened from the plasma membrane to the membrane of the cilium. The Journal of cell biology $187,365-$ 374.

Miranda, M.R., Bouvier, L.A., Canepa, G.E., and Pereira, C.A. (2009). Subcellular localization of Trypanosoma cruzi arginine kinase. Parasitology 136, 1201-1207. Morga, B., and Bastin, P. (2013). Getting to the heart of intraflagellar transport using Trypanosoma and Chlamydomonas models:

the strength is in their differences. Cilia, in press.

Nonaka, S., Tanaka, Y., Okada, Y., Takeda, S., Harada, A., Kanai, Y., Kido, M., and Hirokawa, N. (1998). Randomization of left-right asymmetry due to loss of nodal cilia generating leftward flow of extraembryonic fluid in mice lacking KIF3B motor protein. Cell $95,829-837$.

Oberholzer, M., Langousis, G., Nguyen, H.T., Saada, E.A., Shimogawa, M.M., Jonsson, Z.O., Nguyen, S.M., Wohlschlegel, J.A., and Hill, K.L. (2011). Independent analysis of the 
flagellum surface and matrix proteomes provides insight into flagellum signaling in mammalian-infectious Trypanosoma brucei. Molecular \& cellular proteomics : MCP 10, M111 010538.

Oberholzer, M., Lopez, M.A., Ralston, K.S., and Hill, K.L. (2009). Approaches for functional analysis of flagellar proteins in African trypanosomes. Methods Cell Biol 93, 21-57.

Ooi, C.P., and Bastin, P. (2013). More than meets the eye: understanding Trypanosoma brucei morphology in the tsetse. Frontiers in cellular and infection microbiology 3, 1-12.

Ooi, C.P., Rotureau, B., Gribaldo, S., Georgikou, C., Julkowska, D., Blisnick, T., Perrot, S., Subota, I., and Bastin, P. (2015). The Flagellar Arginine Kinase in Trypanosoma brucei Is Important for Infection in Tsetse Flies. PLoS One 10, e0133676.

Owa, M., Furuta, A., Usukura, J., Arisaka, F., King, S.M., Witman, G.B., Kamiya, R., and Wakabayashi, K. (2014). Cooperative binding of the outer arm-docking complex underlies the regular arrangement of outer arm dynein in the axoneme. Proc Natl Acad Sci U S A 111, 9461-9466.

Pazour, G.J., Agrin, N., Leszyk, J., and Witman, G.B. (2005). Proteomic analysis of a eukaryotic cilium. The Journal of cell biology 170, 103-113.

Piperno, G., Mead, K., and Henderson, S. (1996). Inner dynein arms but not outer dynein arms require the activity of kinesin homologue protein KHP1(FLA10) to reach the distal part of flagella in Chlamydomonas. The Journal of cell biology 133, 371-379.

Portman, N., and Gull, K. (2010). The paraflagellar rod of kinetoplastid parasites: from structure to components and function. International journal for parasitology 40, 135-148. Pradel, L.C., Bonhivers, M., Landrein, N., and Robinson, D.R. (2006). NIMA-related kinase TbNRKC is involved in basal body separation in Trypanosoma brucei. Journal of cell science $119,1852-1863$. 
Ralston, K.S., Kisalu, N.K., and Hill, K.L. (2011). Structure-function analysis of dynein light chain 1 identifies viable motility mutants in bloodstream-form Trypanosoma brucei. Eukaryot Cell 10, 884-894.

Ralston, K.S., Lerner, A.G., Diener, D.R., and Hill, K.L. (2006). Flagellar motility contributes to cytokinesis in Trypanosoma brucei and is modulated by an evolutionarily conserved dynein regulatory system. Eukaryot Cell 5, 696-711.

Reiter, J.F., Blacque, O.E., and Leroux, M.R. (2012). The base of the cilium: roles for transition fibres and the transition zone in ciliary formation, maintenance and compartmentalization. EMBO reports 13, 608-618.

Reiter, J.F., and Leroux, M.R. (2017). Genes and molecular pathways underpinning ciliopathies. Nat Rev Mol Cell Biol.

Robinson, D., Beattie, P., Sherwin, T., and Gull, K. (1991). Microtubules, tubulin, and microtubule-associated proteins of trypanosomes. Methods in enzymology 196, 285-299. Rosenbaum, J.L., Moulder, J.E., and Ringo, D.L. (1969). Flagellar elongation and shortening in Chlamydomonas. The use of cycloheximide and colchicine to study the synthesis and assembly of flagellar proteins. The Journal of cell biology 41, 600-619.

Sapiro, R., Kostetskii, I., Olds-Clarke, P., Gerton, G.L., Radice, G.L., and Strauss, I.J. (2002). Male infertility, impaired sperm motility, and hydrocephalus in mice deficient in spermassociated antigen 6. Mol Cell Biol 22, 6298-6305.

Shen, S., Arhin, G.K., Ullu, E., and Tschudi, C. (2001). In vivo epitope tagging of Trypanosoma brucei genes using a one step PCR-based strategy. Mol Biochem Parasitol 113, 171-173.

Sheriff, O., Lim, L.F., and He, C.Y. (2014). Tracking the biogenesis and inheritance of subpellicular microtubule in Trypanosoma brucei with inducible YFP-alpha-tubulin. BioMed research international $2014,893272$. 
Sherwin, T., and Gull, K. (1989). The cell division cycle of Trypanosoma brucei brucei: timing of event markers and cytoskeletal modulations. Philos Trans R Soc Lond B Biol Sci $323,573-588$.

Smith, E.F., and Lefebvre, P.A. (1996). PF16 encodes a protein with armadillo repeats and localizes to a single microtubule of the central apparatus in Chlamydomonas flagella. The Journal of cell biology 132, 359-370.

Smith, J.C., Northey, J.G., Garg, J., Pearlman, R.E., and Siu, K.W. (2005). Robust method for proteome analysis by MS/MS using an entire translated genome: demonstration on the ciliome of Tetrahymena thermophila. J Proteome Res 4, 909-919.

Song, L., and Dentler, W.L. (2001). Flagellar protein dynamics in Chlamydomonas. The Journal of biological chemistry 276, 29754-29763.

Subota, I., Julkowska, D., Vincensini, L., Reeg, N., Buisson, J., Blisnick, T., Huet, D., Perrot, S., Santi-Rocca, J., Duchateau, M., Hourdel, V., Rousselle, J.C., Cayet, N., Namane, A., Chamot-Rooke, J., and Bastin, P. (2014). Proteomic analysis of intact flagella of procyclic Trypanosoma brucei cells identifies novel flagellar proteins with unique sub-localization and dynamics. Molecular \& cellular proteomics : MCP 13, 1769-1786.

Sunter, J.D., Varga, V., Dean, S., and Gull, K. (2015). A dynamic coordination of flagellum and cytoplasmic cytoskeleton assembly specifies cell morphogenesis in trypanosomes. Journal of cell science $15,1580-1594$.

Vincensini, L., Blisnick, T., and Bastin, P. (2011). 1001 model organisms to study cilia and flagella. Biol Cell 103, 109-130.

Voncken, F., Gao, F., Wadforth, C., Harley, M., and Colasante, C. (2013). The phosphoarginine energy-buffering system of trypanosoma brucei involves multiple arginine kinase isoforms with different subcellular locations. PLoS ONE 8, e65908. 
Wei, Y., Hu, H., Lun, Z.R., and Li, Z. (2014). Centrin3 in trypanosomes maintains the stability of a flagellar inner-arm dynein for cell motility. Nature communications 5, 4060. Wirtz, E., and Clayton, C. (1995). Inducible gene expression in trypanosomes mediated by a prokaryotic repressor. Science 268, 1179-1183.

Wren, K.N., Craft, J.M., Tritschler, D., Schauer, A., Patel, D.K., Smith, E.F., Porter, M.E., Kner, P., and Lechtreck, K.F. (2013). A differential cargo-loading model of ciliary length regulation by IFT. Curr Biol 23, 2463-2471.

Yagi, T., Uematsu, K., Liu, Z., and Kamiya, R. (2009). Identification of dyneins that localize exclusively to the proximal portion of Chlamydomonas flagella. Journal of cell science 122 , 1306-1314.

Ye, F., Breslow, D.K., Koslover, E.F., Spakowitz, A.J., Nelson, W.J., and Nachury, M.V. (2013). Single molecule imaging reveals a major role for diffusion in the exploration of ciliary space by signaling receptors. eLife 2, e00654.

Zhou, Q., Hu, H., He, C.Y., and Li, Z. (2015). Assembly and maintenance of the flagellum attachment zone filament in Trypanosoma brucei. Journal of cell science 128, 2361-2372. 


\section{Figure legends}

\section{Figure 1}

\section{The radial spoke protein RSP3 is incorporated at the distal tip of the flagellum}

(A) Western blot showing inducible expression of Ty1::RSP3 upon tetracycline induction. Total (T), soluble (S) and cytoskeletal (C) protein extracts of non-induced cells $(0 \mathrm{~h})$, and cells induced for $1 \mathrm{~h}$ and $48 \mathrm{~h}$ were prepared. The membrane was incubated with the BB2 monoclonal antibody directed against the Ty1 tag to detect the Ty1::RSP3 fusion protein (top panel), or the anti-BiP as a loading and fractionation control (bottom panel).

(B) In bi-flagellated cells, the new assembling flagellum is posterior to the cell and shorter than the old flagellum in maintenance. Cells were fixed in methanol and stained with the Mab25 antibody to detect the axoneme (red) and with DAPI (blue). F: flagellum, N: nucleus, K: kinetoplast.

(C) 1 h-induced and $4 \mathrm{~h}$-induced $T y 1:: R S P 3$ cells were treated with $1 \%$ NP40 prior to methanol fixation, stained with the Mab25 antibody to detect the axoneme (red, left panels) and the BB2 antibody to detect Ty1::RSP3 (green, middle panels) then counterstained with DAPI (blue). Sites of incorporation of newly synthesised proteins are indicated with long white arrows.

Yellow arrow: new flagellum. White arrow: old flagellum. Scale bar: $5 \mu \mathrm{m}$

\section{Figure 2}

The central pair protein PF16 is incorporated at the distal tip of the flagellum

(A) Western blot showing inducible expression of PF16 upon tetracycline induction. 
Total (T), soluble (S) and cytoskeletal (C) protein extracts of non-induced cells, and cells induced for $1 \mathrm{~h}, 1.5 \mathrm{~h}, 2.5 \mathrm{~h}$ and $48 \mathrm{~h}$ were prepared. The membrane was incubated with the BB2 antibody directed against the Ty1 tag to detect the PF16::Ty1 fusion protein (top panel), the L13D6 antibody to detect the PFR (middle panel) or the anti-BiP (bottom panel), as loading and fractionation controls.

(B) 2.5-hour-induced PF16::Ty1 cells were treated with 1\% NP40 prior to methanol fixation, stained with the Mab25 antibody to detect the axoneme (red, left panel) and the BB2 antibody to detect Ty1::PF16 (green, middle panels) then counterstained with DAPI (blue, left and right panels). The site of incorporation of newly synthesised proteins is indicated with long white arrows. A weak homogenous signal can be seen on the old flagellum and corresponds to a low level of expression of PF16::Ty1 due to a slight leakiness of the inducible system as also observed on the western blot.

Yellow arrow: new flagellum. White arrow: old flagellum. Scale bar: $5 \mu \mathrm{m}$

\section{Figure 3}

\section{The outer dynein arm protein DNAI1 undergoes both distal incorporation and}

\section{intercalation}

FRAP analysis of trypanosomes expressing the GFP::DNAI1 fusion protein. A. Western blot showing expression of GFP::DNAI1. Total (T), soluble (S) and cytoskeletal (C) protein were prepared. The membrane was incubated with the anti-GFP antibody that detects only the GFP::DNAI1 fusion protein (top panel) and then with the anti-DNAI1 antibody that detects both the endogenous DNAI1 protein and the GFP::DNAI1 fusion protein (bottom panel). B. The new flagellum and the proximal part of the old flagellum were bleached with a brief laser pulse (framed area) and recovery was monitored upon acquisition of an image every 3 min, for up to $2 \mathrm{~h}$. Pre-bleach situation: old and new flagella are equally positive for GFP::DNAI1. 
Post-bleach situation: only the second half of the old flagellum remains positive. Recovery of fluorescent signals is shown at the indicated times. Cartoons under each panel show the situation for old (black) and new (purple) flagella. Closed and open segments are GFP positive and negative, respectively. Recovered signal is shown in green.

Yellow arrow: new flagellum. White arrow: old flagellum. Scale bar: $5 \mu \mathrm{m}$

\section{Figure 4}

The outer dynein arm heavy chain B does do not show visible turnover in the mature flagellum

FRAP analysis of trypanosomes expressing the GFP::DHCODAB fusion protein. The distal end of the flagellum was bleached with a brief laser pulse (framed area) and recovery was monitored upon acquisition of a series of 20 images at each of the indicated time points. This is a typical example out of 14 cells from 2 different experiments. A. Pre-bleach situation: the flagellum is positive along its length. Darker areas correspond to regions that are not in the focal plane. B. Post-bleach situation: only the proximal part of the flagellum remains positive. The length of the fluorescent portion has been measured and is indicated at the bottom of each image. C-G. The fluorescent signal is shown at the indicated times. No recovery could be detected in the flagellum. The thin fluorescent portion corresponds to the anterior end of the cell body and not the flagellum. Scale bar: $5 \mu \mathrm{m}$. This is a typical example coming from two series with 8 cells each.

\section{Figure 5}

The flagellum membrane AK3 shows fast and non-polarised incorporation in growing and mature flagella. 
(A) Western blot showing inducible expression of AK3::TY1 upon tetracycline induction. Total protein extracts of non-induced cells, and cells induced for 2 and $4 \mathrm{~h}$ were prepared. The membrane was incubated with the BB2 antibody directed against the Ty1 tag, to detect the AK3::Ty1 fusion protein (bottom panel), the anti-AK antibody (middle panel) and the L13D6 antibody (top panel) to detect the PFR as a loading control.

(B) 30-min-induced $A K 3:: T y 1$ cells were subjected to PFA fixation, and stained with the BB2 antibody to detect Ty1::AK3 (green) then counterstained with DAPI. Cells are shown at different time points of the cell cycle $(1 \mathrm{~K} 1 \mathrm{~N}, 2 \mathrm{~K} 1 \mathrm{~N}, 2 \mathrm{~K} 2 \mathrm{~N})$.

Scale bar: $5 \mu \mathrm{m}$

\section{Figure 6}

\section{Entry and maintenance of AK3 in the flagellum does not rely on IFT}

$I F T 140^{R N A i}$ and $I F T 88^{R N A i}$ cells were grown in the absence of tetracycline (no RNAi, A) or in the presence of tetracycline for $48 \mathrm{~h}(\mathrm{~B}-\mathrm{C})$. Cells were stained with an anti-AK antibody and with the flagellum transition zone component (FTZC), as a marker of the base of the flagellum. The right panel shows the phase contrast image merge with DAPI (cyan) to reveal nuclear and kinetoplast DNA. The left panel shows the immunofluorescence images with the anti-AK (that detects all three AK proteins) in green and the anti-FTZC in red. Arrows indicate remaining flagella in the induced sample that all remain positive for arginine kinase. Note the increase of arginine kinase signal in the cytoplasm in induced samples. Scale bar: $5 \mu \mathrm{m}$.

\section{Supplementary figure 1}

Outer dynein arm intermediate chain DNAI1 does not show visible turnover in the mature flagellum 
FRAP analysis of trypanosomes expressing the GFP::DNAI1 fusion protein. The distal end of the mature flagellum was bleached with a brief laser pulse (framed area) and recovery was monitored upon acquisition at the indicated time points (one typical example out of 5 videos). A. Pre-bleach situation: the flagellum is positive along its length. B. Post-bleach situation: only the proximal part of the flagellum remains positive. The length of the fluorescent portion has been measured and is indicated at the bottom of each image. C-G. The fluorescent signal is shown at the indicated times. No recovery could be detected in the distal portion of the flagellum. Scale bar: $5 \mu \mathrm{m}$. This is a typical example coming from two series with 3 cells each. 


\section{Graphical abstract}

Cilia and flagella are composed of hundreds of different proteins found in a sophisticated skeletal structure called axoneme, in the matrix and in the membrane. Incorporation of new skeletal components takes place at the distal end of growing flagella and shows little or no turnover in mature flagella. In contrast, the membrane-associated protein arginine kinase 3 (AK3) displays rapid turnover without a specific polarity in both mature and growing flagella. 